\title{
Gut Microbiome and Social Determinants of Health (SDOH)
}

Suneeta Kumari MD, MPH*, Saba Afzal MD, Stacy Doumas MD, Eric Alcera MD, Ramon Solhkhah MD

Hackensack Meridian Health- Ocean Medical Center- Hackensack Meridian School of Medicine, Brick NJ.

*Corresponding Author: Suneeta Kumari, Hackensack Meridian Health- Ocean Medical Center- Hackensack Meridian School of Medicine, Brick NJ.

Received date: September 16, 2020; Accepted date: September 30, 2020; Published date: November 05, 2020

Citation: S Kumari, S Afzal, S Doumas, E Alcera, R Solhkhah. (2020) Gut Microbiome and Social Determinants of Health (SDOH). International Journal of Clinical Case Reports and Reviews. 4(2); DOI: 10.31579/2690-4861/060

Copyright: ( 2020 Suneeta Kumari, This is an open-access article distributed under the terms of the Creative Commons Attribution License, which permits unrestricted use, distribution, and reproduction in any medium, provided the original author and source are credited.

\begin{abstract}
With technological advancements in the medical field, new discoveries have been unfolded about the human microbiota. A tremendous amount of work has been studied within the last two decades. Some of the human microbiota sites include nonsterile areas such as mouth, skin, gut, nose, and vagina. Additionally, there are bacterial cells in areas that were considered sterile such as lungs and placenta before delivery. Out of all the sites, the gut houses the most with an amount of 100 trillion bacteria (Guinane, 2013). Environmental implications have been known to impact these new areas of medicine. There has been a growing interest by the social epidemiologists on how health inequalities impact the role of human gut microbiota.
\end{abstract}

Keywords: Gut Microbiome; SDOH; human microbiota

\section{Introduction}

With technological advancements in the medical field, new discoveries have been unfolded about the human microbiota. A tremendous amount of work has been studied within the last two decades. Some of the human microbiota sites include nonsterile areas such as mouth, skin, gut, nose, and vagina. Additionally, there are bacterial cells in areas that were considered sterile such as lungs and placenta before delivery. Out of all the sites, the gut houses the most with an amount of 100 trillion bacteria (Guinane, 2013). Environmental implications have been known to impact these new areas of medicine. There has been a growing interest by the social epidemiologists on how health inequalities impact the role of human gut microbiota.

The gut microbiome has been linked to multiple behavioral health conditions, including depression, PTSD, anxiety, and bipolar disorder. Like other health conditions, a broader social environment shapes the microbiome over the life course. The gut microbiome is influenced by social disparities. Research shows that environmental factors have a greater impact on gut microbiota compared to genetics. There has been a significant resemblance in the composition of the microbiomes of genetically unrelated individuals who live together (Rothschild, 2018).

In this article, authors aimed to explore how early development in life, socioeconomic inequalities, social stressors, and health behaviors can impact the human intestinal microbiome. This review attempts to highlight the scope and mechanism of these influences and how the social environment can influence the human gut microbiota.

There is a growing awareness and interest that gut microbiota can play a crucial role in maintaining homeostasis in health and contribute to the pathogenesis of variety of diseases including disorders of CNS. Several studies have shown role of gut microbiota to influence gut-brain communication in health and disease (Dinan, 2013, Stantan 2017).
Keywords: human microbiome, gut microbiome, social determinants of health, socioeconomic inequalities, health disparities, early development, social stressors, health behavior

\section{Understanding gut-brain axis}

The gut- brain axis is a bidirectional communication system through which the brain modulates/ regulates GI function. In this process several neural, endocrine, and immunological mechanisms play important/ essential role. The intestinal microbiota impacts the GI physiology, including the development and function of enteric nervous system- Also known as second brain- It controls the GI function independently. The enteric system of GI is composed of myenteric and submucosal plexus. Interestingly, recent research findings suggest a potential link of these structures in neurodegenerative disorders- For instance characteristic lewy bodies, pathological hallmarks of Parkinson's disease, were found in intestinal biopsies of patients with Parkinson's disease (Lebouvier 2009).

There is increasing evidence that the immune system, inflammation and mucosal barrier function are involved in the pathogenesis of some psychiatric diseases. For instance-in depression, "leaky gut" has been suggested to play a significant pathogenic role- This assumption was based on findings of prevalence and median values for serum $\operatorname{IgM}$ and IgA against lipopolysaccharide of enterobacteria in patients with MDD then in normal volunteers (Maes M. Kubera 2008). This study suggested that patient with MDD should be checked for leaky gut by means of $\operatorname{IgM}$ and IgA panels and should be treated for leaky gut accordingly.

\section{Effect of gut microbiota on the CNS.}

- Gut microbiome is an integral part of Gut-Brain axis. The interaction between gut microbiome, gut permeability and CNS is BIDIRECTIONAL. (Yarandi 2016) 
- Presence of healthy and diverse gut microbiota is important to normal cognitive and emotional processing. Chronic stress can change composition of gut microbiome.

- Alteration in composition of gut microbiome DUE TO STRESS can lead to increased intestinal permeability--- lead to translocation of gut microbiota and metabolic product such as lipopolysaccharides through the intestinal barrier.
- Subsequently neuroactive compound gain access to the CNS that regulates cognition and emotional responses.

- Stress can lead to activation of the hypothamus -pitutary axis and excessive release of corticotropin-releasing factor (CRH). This hormone along with altered vagal activity can cause local activation of mast cells in the intestinal wall and release of CYTOKINES, causing increased gut permeability.

- Exposure of epithelial cells or mucosal immune cell to bacteria or metabolic products leads to activation of immune response and release of pro-inflammatory CYTOKINES

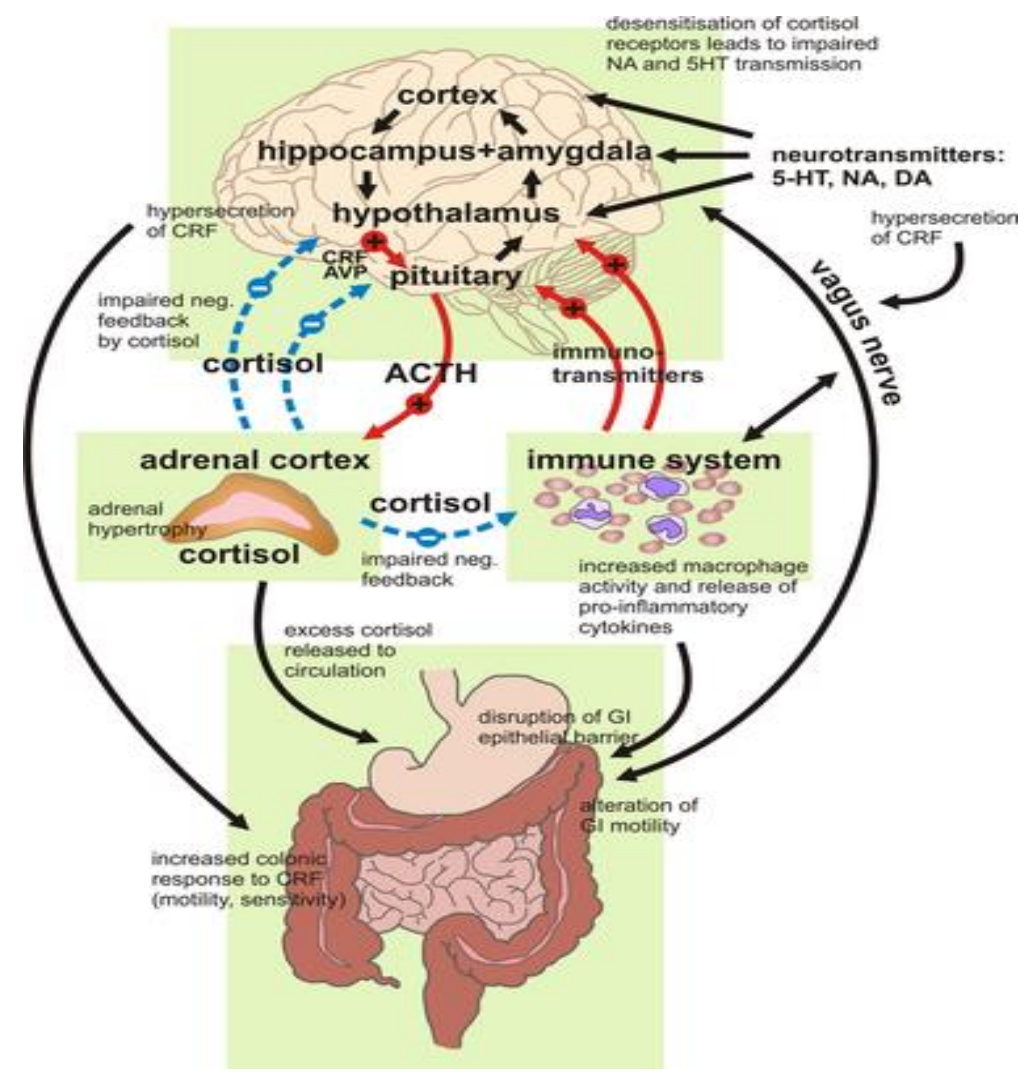

Figure 1: A schematic representation of the effects of chronic stress and depression on brain-gut axis activity. The bi-directional communication allows signals from the brain corticolimbic structures to alter gastrointestinal function. The HPA axis and immune system are key regulators of this axis

\section{Gut Microbiota and Depression}

According to research on animal model- Depression changes the composition of gut microbiota (Park AJ Collins 2013). These data have not been validated in patient with depression. Recent study (Naseribafrouei 2014) examined the composition of fecal microbiota in 46 patients with depression and 30 healthy controls. This study reported significant differences with increased population of Bacteriodetes, Proteobacteria, Antinibacteria and decreased population of Frimicutes in patients with depression. Other evidence that might suggest role of gut microbiota in the pathogensis of depression is from studies that have shown certain probiotics can alleviate depressive symptoms in rodent models (Park AJ Collins 2013).

According to Dinan 2013 study- A variety of strategies have been used to study the impact of the microbiota on brain function and these include antibiotic use, probiotic treatments, fecal microbiota transplantation, gastrointestinal infection studies, and germ-free studies. All these approaches provide evidence to support the view that the microbiota can influence brain chemistry and consequently behavior (Dinan 2013).

Additionally, research from animal demonstrate that there is a distinct perturbation of the composition of gut microbiota in animal models of depression and chronic stress. This has direct implications for the development of psychobiotic-based therapeutic strategies for psychiatric disorders. Moreover, given that affective co-morbidities, such as major depression and anxiety states, are common in patients presenting with irritable bowel syndrome (IBS), it may have implications for functional bowel disorders as well.

\section{Role of psychobiotic/ probiotics:}

Normal gut microbiota is essential in preventing colonization of the harmful bacteria. In the absence of normal flora (antibiotic therapy)pathogenic organisms produce toxins and colonize the gut epithelium (C. Difficile). Probiotic treatment-reduces gut permeability, enhance mucus production, improve physical barrier protecting the epithelial layer 
(Yarandi 2016) Treatment of rats with probiotics containing B. infantis can reduce the mood disturbance and correct the concentration on norepi (NE) in the brain (Desbonnet L, 2010). In a model of depression post MI, treatment with probiotics reduce the depression, presumably by reducing the pro-inflammatory cytokines and gut permeability (Arseneault-Breard 2012)

Previous research defines a psychobiotic as a live organism that, when ingested in adequate amounts, produces a health benefit in patients suffering from psychiatric illness. As a class of probiotic, these bacteria can produce and delivering neuroactive substances such as gammaaminobutyric acid (GABA) and serotonin, which act on the brain-gut axis (Dinen 2013). Preclinical evaluation in rodents suggests that certain psychobiotics possess antidepressant or anxiolytic activity. Effects may be mediated via the vagus nerve, spinal cord, or neuroendocrine systems (Dinen 2017).

To date, psychobiotics have been most extensively studied in a liaison psychiatric setting in patients with irritable bowel syndrome, where positive benefits have been reported for several organisms including Bifidobacterium infantis. Evidence is emerging of benefits in alleviating symptoms of depression. Such benefits may be related to the antiinflammatory actions of certain psychobiotics and a capacity to reduce hypothalamic-pituitary-adrenal axis activity. Results from large scale placebo-controlled studies are awaited.

In one UK study, 13,000 male and female twins within the age group of 18 and 103 studied sociodemographic and health variables that were matched with microbiota from a previous sample study. This was analyzed using $16 \mathrm{~S}$ sequence. Socioeconomic status was measured using the Index of Multiple Deprivation 2015 which encompassed income, employment, education, skills and training, health deprivation and disability, crime, barriers to housing and services, and living environment deprivation based on the postcode. These measures were taken from the Scottish and English/Welsh datasets from the Scottish Government and Public Health England. The microbiota composition was measured in relation to alpha diversity, beta diversity, and differential operational taxonomic units (Bowyer, 2019).

\section{Early Development}

From early on in development, the various components of social determinants of health can affect the individuals' gut microbiome. The first few years of life play a critical role in the development of a child. These early stages of gut microbiota can have a significant impact later in life. One of many factors where gut microbiota difference can be explained is starting with the mode of delivery of a fetus. In the United States, there has been a rise in C-section deliveries from $21 \%$ in 1997 to $33 \%$ in 2011 (Witt, 2015). A study observed that C-section infants had a reduced number of bacteria and decreased resemblance to their mothers in comparison to infants born vaginally (Roswell, 2015). Additionally, breast-fed infants had high-quality gut microbiota such as B. longum compared to formula-fed infants which had poor types of bacteria such as C. difficile (Dowd, 2018).

Furthermore, the transition from breastmilk to solid foods diversifies the microbiome. Compared to healthy children, those who were malnourished showed delayed microbiome. Psychological stressors early in life can impact the gut microbiota. Infant monkeys separated from their mothers created physiological stress that showed a great reduction in the number of Lactobacilli from the stool (Galley, 2014). Early healthcare experiences can also contribute to the gut microbiota. For instance, inappropriate use of antibiotics given to children in the first few years of life have shown unstable microbiome compared to healthy counterpart (Knight, 2017). Children commonly get viral infections early in life, and it is detrimental to the child's gut when treated with antibiotics. In the
USA, non-Hispanic Caucasian children seem to receive more antibiotics for a viral infection compared to other races (Goyal, 2017). Contrastingly, African American children were less likely to receive antibiotics for their bacterial infection (Gerber, 2013). This shows the discrepancy of how privileged groups were able to fulfill their needs, whereas minority groups had difficulty accessing the medical care that they needed. Therefore, several environmental factors early in life such as mode of delivery, feeding methods, psychological stress, and inappropriate use of antibiotics can shape the gut microbiome.

\section{Effects of the Socioeconomic Inequalities on Mental Health}

Although there are studies that have heavily investigated the relationship between socioeconomic inequality and mortality from various health conditions, there is a limitation on the understanding of the biological mechanism. To explain the biological side, some studies have shown that there is a relationship between neighborhood socioeconomic status with the alpha diversity of the gut microbiota (Miller, 2016). Alpha diversity provides the quality of richness in the microbiota of an individual. Therefore, lower socioeconomic status has been associated with reduced alpha diversity of the gut microbiota. Specifically, those with lower incomes showed a reduction in alpha diversity. When there is a reduced amount of alpha diversity, it is common to see a negative effect on health status (Bowyer, 2019).

Furthermore, the reduced alpha diversity of the microbiota has been found to contribute to psychiatric disorders. For instance, a study transplanted the microbiota of individuals with major depressive disorder into germfree mice that resulted in these mice to encounter depression-like symptoms. This study explained that the depression microbiota showed disturbances in microbiota genes and metabolites associated with carbohydrate and amino acid metabolism (Zheng, 2016). Other studies have shown reduced alpha diversity with ADHD, autism, and anxiety disorders. Therefore, studies have identified that probiotic treatment can have possible antidepressant properties to help those with depression-like behaviors and other mental health-related conditions.

\section{Social stressors}

More recently, there has been some study where stressors and other factors can directly influence the gut microbiota. Some studies have shown that gut microbiota has an involvement in the way the brain function through the 'microbiota-gut-brain axis'. This terminology defines the bidirectional communication network as discussed above that involves the gut functions with several factors from the central nervous system, autonomic nervous system, enteric nervous system, and the hypothalamic-pituitary-adrenal axis (Carabotti, 2015). This regulation can explain how the decrease in diversity of the gut microbiota can impact the brain's serotonergic and neuroendocrine systems. For instance, norepinephrine contributes to the stimulation of the growth in E. coli of the intestinal tract during a traumatic event (Freestone, 2002). In mice, consistent exposure to social stressors resulted in changes to the microbiota immune system where infection became more likely along with inflammatory mediators (Bailey, 2014).

There is evidence that suggests immunological implications are associated with the pathophysiology of psychiatric disorders. One study examined that the intestinal mucosal dysfunction (leaky gut) can be associated with major depressive disorder due to an inflammatory response (Maes, 2008). In humans, there was a greater alpha diversity in the gut microbiota of 73 soldiers before and after stress-inducing military environment. This study showed over $50 \%$ abundance in $16 \mathrm{~S}$ taxa classified from stool samples (Karl, 2017).

In another study with 23 college students, there was a reduction in the number of Lactobacilli when students experienced a high level of stress 
for multiple days during final exams (Knowles, 2007). Although there are limited studies involving humans, these studies show how social implications have a tremendous factor in the gut microbiota. It will be interesting to see how sensitive the gut microbiota can be with daily stressors. The ones mentioned are more stressful events in a limited amount of time, but another area to consider is to see how those types of stressors impact the gut microbiota for a longer time frame.

\section{Behavioral Health Changes}

Behavioral health differences can influence the changes between socioeconomic factors and gut microbiota. For instance, diet, smoking, and pharmacological agents can all play into the connections between the gut microbiota and social status.

\section{Diet}

As in the early stages of life with feeding practices, the foods that we consume as adults have great effects on the makeup of our gut microbiome. Implementing changes to the diet show the alterations of the microbiota composition within 24 hours and in longer-term (Wu, 2011). Diets with high in animal fat and protein have shown to reduce A. muciniphila and Lactobacillus which have been noted to be associated with healthy metabolic states. These changes have been linked with inflammation, decrease insulin sensitivity, and an increase in LDL cholesterol. On the other hand, diets rich in non-digestible carbohydrates such as fiber and resistant starch have been shown to consistently increase intestinal Bifidobacteria and Lactobacilli which showed a reduction in pro-inflammatory cytokine IL-6, insulin resistance, LDL-cholesterol and hemoglobin A1c (Singh, 2017). The socioeconomic factors within race and ethnicity provide information that there are differences with diet. There have been findings that non-Hispanic African American adults consume less dietary fiber compared to other races and ethnic groups. Furthermore, lower-income families who are living at less than $131 \%$ of poverty have been associated with lower dietary fiber consumption (Storey, 2014).

\section{Smoking}

In both, animal and human studies have shown that tobacco alters the composition of the microbiome. In mice studies, smoking changes the gut microbiota shown to create an inflammatory microenvironment in the intestines. This inflammatory condition has been supported through several studies showing that smoking increases Clostridium, Bacteroides and Prevotella, and decreases Bifidobacteria and Lactobacilli. Additionally, changes in the intestinal microbiome from smoking showed similar conditions in inflammatory bowel disease and obesity (Savin, 2018). In human studies, there may be a promising indication that smoking alterations of the oral microbiome could be reversible considering that former smokers and never smokers did not differ in their microbiome composition (Wu, 2016).

\section{Pharmacological Agents}

The topic of social factors to prescription medication in relation to alteration of gut microbiota is still limited in evidence. Mentioned in the early development, the inappropriate use of antibiotics is detrimental to the gut microbiota in adults as well which can cause short-term and longterm effects. Antibiotic use decreases the diversity and changes the composition of the gut microbiota which increases the risk of disease, secondary infections, allergies, and obesity (Becattini, 2016). Besides antibiotics, other medications such as antipsychotics, opioids, metformin, statins, proton pump inhibitors, nonsteroidal anti-inflammatory drugs can all contribute to the alterations of the gut microbiome. Antipsychotic and proton pump inhibitors have been noted to decrease the alpha diversity in the gut microbiota, and contrastingly, opioids showed an increase in alpha diversity (Bastard, 2017). Opioids have been shown to be prescribed more frequently in emergency departments for Caucasians and those with higher socioeconomic status. African American and Hispanic patients were noted to receive fewer opioids for equivalent levels of pain compared to Caucasians (Joynt, 2013). For the others, there is not enough data to conclude how social variation influence the use of these medications.

\section{Discussions}

With more research in the human microbiome, studies have been able to see associations between the intestinal microbiome and the social determinants of health. Various exposures early in life, socioeconomic inequalities related to mental health, social stressors, and health behaviors are some of the factors that influence the gut microbiota. Common findings among several studies showed that a decrease in Lactobacilli can increase inflammations and can lead to risk factors for different types of diseases. Other common findings presented with those in the lowerincome population had reduced diversity in their gut microbiome. There are many layers that may explain this phenomenon for the underserved community, where lack of access to produce and medical care can contribute to the reduction of certain healthy bacteria.

Since various exposures can alter the intestinal composition of the microbiota, health conditions could be better understood by getting a fecal sample on disorders with limited information. For example, knowing how social variations impact the gut microbiota in relation to behavioral disorders can better provide clinicians with treatment plans tailored to their patient's needs. Since various environmental changes influence the gut microbiota, it will be interesting to see in future studies how reversible gut microbiota composition can possibly improve the quality of the patient's health.

Areas of improvement in studies between gut microbiota and social determinants of health include conducting more human models research. Most of the literature linking the social environment and the microbiome comes from animal models. These models usually focus on the impact of social interactions and psychosocial stress. Other important factors in humans may include the importance of early life exposures to stress, vulnerability and health behaviors.

Another area for exploration includes the implementation of probiotics and how much this can change the gut microbiome. Some studies have been able to observe the antidepressant properties in probiotics, but more research could be conducted to better support this information. This can be a promising treatment for those who cannot have access to affordable prescription medications when going through treatment resistant mental disorders.

Lastly, there is a cyclic pattern that can be identified where social variation influences gut microbiota which can lead to various risk factors. Social epidemiologists can continue to explore more studies pertaining to the connection between gut microbiome and social determinants of health to help clinicians better understand some of the health conditions, especially in behavioral disorders. Overall, this can provide improvements in diagnosis and treatment options for the patients.

\section{Summary}

Ultimately, what is at stake here is that several social conditions can affect the gut microbiome by both the upstream and downstream mechanisms. As population-level microbiome data becomes increasingly available, we hope that multi-level determinants of the gut microbiome will become clearer. There is a greater need to further elucidate the mechanisms by which microbiome may link the social environment and health.

Acknowledgement: The authors like to thank Vinod Kumar, Shivam Chand and Vinay Kumar for their assistance with literature search, editing and proofreading of this review article. 


\section{References}

1. Bäckhed, F., Roswall, J., Peng, Y., Feng, Q., Jia, H., Kovatcheva-Datchary, P., Li, Y., Xia, Y., Xie, H., Zhong, H., Khan, M. T., Zhang, J., Li, J., Xiao, L., Al-Aama, J., Zhang, D., Lee, Y. S., Kotowska, D., Colding, C., ... Jun, W. (2015). Dynamics and Stabilization of the Human Gut Microbiome during the First Year of Life. Cell Host \& Microbe, 17(5), 690703.

2. Bailey, M. T. (2014). Influence of Stressor-Induced Nervous System Activation on the Intestinal Microbiota and the Importance for Immunomodulation. In M. Lyte \& J. F. Cryan (Eds.), Microbial Endocrinology: The Microbiota-Gut-Brain Axis in Health and Disease (pp. 255-276). Springer.

3. Becattini, S., Taur, Y., \& Pamer, E. G. (2016). AntibioticInduced Changes in the Intestinal Microbiota and Disease. Trends in Molecular Medicine, 22(6), 458-478.

4. Bowyer, R. C. E., Jackson, M. A., Le Roy, C. I., Ni Lochlainn, M., Spector, T. D., Dowd, J. B., \& Steves, C. J. (2019). Socioeconomic Status and the Gut Microbiome: A TwinsUK Cohort Study. Microorganisms, 7(1), 17.

5. Carabotti, M., Scirocco, A., Maselli, M. A., \& Severi, C. (2015). The gut-brain axis: Interactions between enteric microbiota, central and enteric nervous systems. Annals of Gastroenterology: Quarterly Publication of the Hellenic Society of Gastroenterology, 28(2), 203-209.

6. Changes in intestinal microbiota composition and metabolism coincide with increased intestinal permeability in young adults under prolonged physiological stress | American Journal of Physiology-Gastrointestinal and Liver Physiology. (n.d.). Retrieved June 2, 2020.

7. Cigarette smoking and the oral microbiome in a large study of American adults | The ISME Journal. (n.d.). Retrieved June 2, 2020.

8. Desbonnet, L., Garrett, L., Clarke, G., Bienenstock, J., \& Dinan, T. G. (2008). The probiotic Bifidobacteria infantis: An assessment of potential antidepressant properties in the rat. Journal of Psychiatric Research, 43(2), 164-174.

9. Dowd, J. B., \& Renson, A. (2018). "Under the Skin" and into the Gut: Social Epidemiology of the Microbiome. Current Epidemiology Reports, 5(4), 432-441.

10. Freestone, P. P., Williams, P. H., Haigh, R. D., Maggs, A. F., Neal, C. P., \& Lyte, M. (2002). Growth stimulation of intestinal commensal Escherichia coli by catecholamines: A possible contributory factor in trauma-induced sepsis. Shock (Augusta, Ga.), 18(5), 465-470.

11. Galley, J. D., Nelson, M. C., Yu, Z., Dowd, S. E., Walter, J., Kumar, P. S., Lyte, M., \& Bailey, M. T. (2014). Exposure to a social stressor disrupts the community structure of the colonic mucosa-associated microbiota. BMC Microbiology, 14(1), 189.

12. Gerber, J. S., Prasad, P. A., Localio, A. R., Fiks, A. G., Grundmeier, R. W., Bell, L. M., Wasserman, R. C., Rubin, D. M., Keren, R., \& Zaoutis, T. E. (2013). Racial Differences in Antibiotic Prescribing by Primary Care Pediatricians. Pediatrics, 131(4), 677-684.

13. Goyal, M. K., Johnson, T. J., Chamberlain, J. M., Casper, T. C., Simmons, T., Alessandrini, E. A., Bajaj, L., Grundmeier, R. W., Gerber, J. S., Lorch, S. A., Alpern, E. R., \& Network (PECARN), for T. P. C. A. R. (2017). Racial and Ethnic Differences in Antibiotic Use for Viral Illness in Emergency Departments. Pediatrics, 140(4).

14. Guinane, C. M., \& Cotter, P. D. (2013). Role of the gut microbiota in health and chronic gastrointestinal disease:
Understanding a hidden metabolic organ. Therapeutic Advances in Gastroenterology, 6(4), 295-308.

15. Joynt, M., Train, M. K., Robbins, B. W., Halterman, J. S., Caiola, E., \& Fortuna, R. J. (2013). The Impact of Neighborhood Socioeconomic Status and Race on the Prescribing of Opioids in Emergency Departments throughout the United States. Journal of General Internal Medicine, 28(12), 1604-1610.

16. Knowles, S. R., Nelson, E. A., \& Palombo, E. A. (2008). Investigating the role of perceived stress on bacterial flora activity and salivary cortisol secretion: A possible mechanism underlying susceptibility to illness. Biological Psychology.

17. Maes, M., Kubera, M., \& Leunis, J.-C. (2008). The gut-brain barrier in major depression: Intestinal mucosal dysfunction with an increased translocation of LPS from gram negative enterobacteria (leaky gut) plays a role in the inflammatory pathophysiology of depression. Neuro Endocrinology Letters, 29(1), 117-124.

18. Miller, G. E., Engen, P. A., Gillevet, P. M., Shaikh, M., Sikaroodi, M., Forsyth, C. B., Mutlu, E., \& Keshavarzian, A. (2016). Lower Neighborhood Socioeconomic Status Associated with Reduced Diversity of the Colonic Microbiota in Healthy Adults. PloS One, 11(2), e0148952.

19. Prehn-Kristensen, A., Zimmermann, A., Tittmann, L., Lieb, W., Schreiber, S., Baving, L., \& Fischer, A. (2018). Reduced microbiome alpha diversity in young patients with ADHD. PLoS ONE, 13(7).

20. Rothschild, D., Weissbrod, O., Barkan, E., Kurilshikov, A., Korem, T., Zeevi, D., Costea, P. I., Godneva, A., Kalka, I. N., Bar, N., Shilo, S., Lador, D., Vila, A. V., Zmora, N., PevsnerFischer, M., Israeli, D., Kosower, N., Malka, G., Wolf, B. C., ... Segal, E. (2018). Environment dominates over host genetics in shaping human gut microbiota. Nature, 555(7695), 210-215.

21. Singh, R. K., Chang, H.-W., Yan, D., Lee, K. M., Ucmak, D., Wong, K., Abrouk, M., Farahnik, B., Nakamura, M., Zhu, T. H., Bhutani, T., \& Liao, W. (2017). Influence of diet on the gut microbiome and implications for human health. Journal of Translational Medicine, 15(1), 73.

22. Smoking and the intestinal microbiome $\mid$ SpringerLink. (n.d.). Retrieved June 2, 2020.

23. Systematic review: Human gut dysbiosis induced by nonantibiotic prescription medications - Le Bastard-2018Alimentary Pharmacology \&amp; Therapeutics-Wiley Online Library. (n.d.). Retrieved June 2, 2020.

24. Witt, W. P., Wisk, L. E., Cheng, E. R., Mandell, K., Chatterjee, D., Wakeel, F., Godecker, A. L., \& Zarak, D. (2015). Determinants of Cesarean Delivery in the U.S.: A Lifecourse Approach. Maternal and Child Health Journal, 19(1), 84-93.

25. Wu, G. D., Chen, J., Hoffmann, C., Bittinger, K., Chen, Y.-Y., Keilbaugh, S. A., Bewtra, M., Knights, D., Walters, W. A., Knight, R., Sinha, R., Gilroy, E., Gupta, K., Baldassano, R., Nessel, L., Li, H., Bushman, F. D., \& Lewis, J. D. (2011). Linking long-term dietary patterns with gut microbial enterotypes. Science (New York, N.Y.), 334(6052), 105-108.

26. Zheng, P., Zeng, B., Zhou, C., Liu, M., Fang, Z., Xu, X., Zeng, L., Chen, J., Fan, S., Du, X., Zhang, X., Yang, D., Yang, Y., Meng, H., Li, W., Melgiri, N. D., Licinio, J., Wei, H., \& Xie, P. (2016). Gut microbiome remodeling induces depressive-like behaviors through a pathway mediated by the host's metabolism. Molecular Psychiatry, 21(6), 786-796. 